\title{
EVIDENCE FOR PROTOSTELLAR COLLAPSE IN B335
}

\author{
S. ZHOU \\ Department of Astronomy, University of Ilinois, Urbana, IL 61801
}

\section{N. J. EVANS II}

Department of Astronomy, University of Texas, Austin, TX 78712

C. KÖMPE

Universität-sternwarte Jena, Schillergässchen 2, 6900 Jena, GERMANY

C. M. WALMSLEY

MPI für Radioastronomie, Auf dem Hügel 69, D-5300 Bonn, GERMANY

ABSTRACT We summarize the evidence for protostellar collapse in B335.

B335 is an isolated Bok globule at a distance of 250 pc. It contains a source of far-infrared and submillimeter emission, which drives a well-collimated outflow. Using VLA observations of the $\mathrm{H}_{2} \mathrm{CO} 6 \mathrm{~cm}$ line, we have derived a density distribution of $r^{-\alpha}(1.5<\alpha<2.0)$ for the central $4^{\prime}$ of B335 (Zhou et al. 1990). Given such a density distribution, the inner core regions should be unstable against gravity. Using the CSO $10 \mathrm{~m}$ and the IRAM $30 \mathrm{~m}$ telescopes, we observed B335 in CS J $=2 \rightarrow 1,3 \rightarrow 2$, and $5 \rightarrow 4, \mathrm{H}_{2} \mathrm{CO} \mathrm{J}_{K_{-1} K_{1}}=2_{12} \rightarrow 1_{11}$ and $3_{12} \rightarrow 2_{11}$ and $\mathrm{HCO}^{+} \mathrm{J}=3 \rightarrow 2$. All these lines are wider in the center of $\mathrm{B} 335$ than expected from a static, microturbulent core, indicating increased motion in the inner core region. All the line profiles are well fitted by a model of a collapsing dense core, as exemplified in Fig. 1a for the two $\mathrm{H}_{2} \mathrm{CO}$ lines. Meanwhile, we have ruled out simple alternate models, such as foreground absorption, expansion, rotation, or outflow, based on quantitative arguments as well as models. The details of the observations and analysis are present in a paper by Zhou et al. (1993).

The basic signature of collapse, an asymmetric line profile with stronger blue-shifted emission, can be derived from three basic assumptions: (1) spherical symmetry; $(2)$ density $(n)$ decreases with increasing radius $(r) ;(3)$ the collapse velocity $(v)$ decreases with increasing $r$. Fig. 1b shows a schematic diagram of a collapsing core with the oval curves tracing points with the same projected velocity. Along a light of sight through the infall region, red-shifted emission (at $+V$ relative to the rest velocity of the cloud) comes from gas parcels near points $R_{1}$ and $R_{2}$, and blue-shifted emission at $-V$ from $B_{1}$ and $B_{2}$. If $T_{1}$ and $\tau_{1}$ are the excitation temperature and optical depth for the gas parcels at $R_{1}$ and $B_{1}$, and $T_{2}$ and $\tau_{2}$, for gas parcels at $R_{2}$ and $B_{2}$, the difference between 
the emergent temperatures of red-shifted emission $T_{R}$ and blue-shifted emission $T_{B}$ is

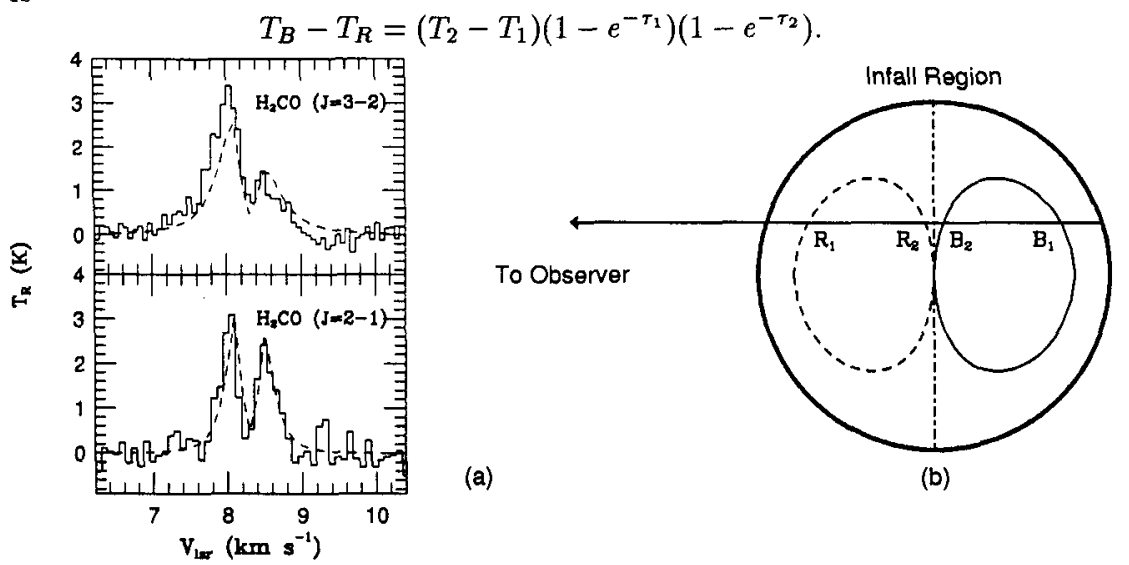

Fig. 1. (a) shows the observed spectra of two $\mathrm{H}_{2} \mathrm{CO}$ lines (histograms) toward the center of $\mathrm{B} 335$ along with model line profiles predicted by a collapse model (dashed lines). (b) shows a schematic diagram of a dense core undergoing inside-out collapse. The oval lines show points with equal projected velocities along the line of sight to the observer.

For transitions with high critical density, we know $T_{2}>T_{1}$ from assumption (2); hence, $T_{B}>T_{R}$. The above formula explains the basic features of Fig. 1a. First, the absorption dips mark the rest velocity of the cloud. Second, the blue-shifted emission is stronger than red-shifted emission. Third, the difference between red-shifted and blue-shifted emission is larger in the $225 \mathrm{GHz}$ line than in the $140 \mathrm{GHz}$ line because the optical depth of the latter line is smaller in the infall region. This dependence of asymmetry in line profiles on the optical depth is the key to ruling out alternate models. It is fairly easy to build a model which produces asymmetric line profiles, but much more difficult to reproduce the dependence of asymmetry on the optical depth.

From the above discussion, we see that the observed spectral features will result if dense cores establish a highly concentrated density distribution prior to collapse and if the collapse velocity is higher in the center. It does not matter whether the initial density distribution is $r^{-2}$ or $r^{-1.5}$. Thus, the spectroscopic evidence for collapse depends only on the outline of the theory of Shu et al. (1987), not on the details. On the other hand, we have ruled out simple alternative models, but a more complex model might be contrived to fit the data. In that case, we would appeal to Ockham's razor, which enjoins us to favor the simplest possible model (see Jefferys and Berger 1992).

\section{REFERENCES}

Jefferys, W.H. \& Berger, J.O. 1992, American Scientist, 80, 64.

Shu, F.H., Adams, F.C., \& Lizano, S. 1987, ARAA, 25, 23.

Zhou, S., Evans, N.J. II, Butner, H.M., Kutner, M.L., Leung, C.M., \& Mundy, L.G. 1990, ApJ, 363, 168 (Paper I).

Zhou, S., Evans, N.J. II, Kömpe, C., and Wlamsley, C.M. 1993, ApJ, in press. 